\title{
MULTI-ROBOT DECENTRALIZED EXPLORATION USING A TRADE-BASED APPROACH
}

\author{
Zhi Yan, Nicolas Jouandeau and Arab Ali Cherif \\ Advanced Computing Laboratory of Saint-Denis (LIASD), Paris 8 University \\ 2 Rue de la Liberté, 93526 Saint-Denis, France \\ yz@ai.univ-paris8.fr,n@ai.univ-paris8.fr,aa@ai.univ-paris8.fr
}

Keywords: Coordinated exploration, Decentralized decision making, Multi-robot systems.

\begin{abstract}
This paper addresses the problem of exploring an unknown environment by a coordinated team of robots. An important question is, which robot should explore which region? In this paper, we present a novel decentralized task allocation approach based on trading rules for multi-robot exploration. In the decentralized system, robots can make their own decisions according to the local information with limited communication. In contrast to previous approaches, our trade-based approach is designed to simulate the relationship between buyers and sellers in a business system, to achieve dynamic task allocation by using a mechanism of unsolicited bid. Our approach has been implemented and evaluated in simulation. The experimental results demonstrate a good performance of the proposed trade-based approach compared to previous approaches.
\end{abstract}

\section{INTRODUCTION}

Exploration of an unknown environment is a fundamental problem in robotics. It requires an agent to cover the unknown area while building a model of the environment from sensor data so as to achieve the purpose of exploration. This research can be used in a wide range of applications such as planetary mission, automated surveillance, and search and rescue operations.

Compared with single agent, using multiple agents has a number of potential advantages (Cao et al., 1997), (Dudek et al., 1996). For example, a team of robots is able to complete an exploration mission faster than a single robot. The key to gain the advantages is coordination. Without coordination, it will not only lower mission efficiency, but also lead to the failure of the entire mission in extreme cases. The core of coordination is task and role allocation. In other words, we need to answer such a question: which agent should execute which task (Gerkey and Matarić, 2004)? Figure 1 shows an example of multiagent coordination. Four robots explore an unknown environment cooperatively. The result of task allocation is that different robots are responsible for exploring different rooms. There are generally two types of mechanisms for task allocation, centralized and decentralized. The advantage of centralized mechanism is that the optimal plans can be found. Never-

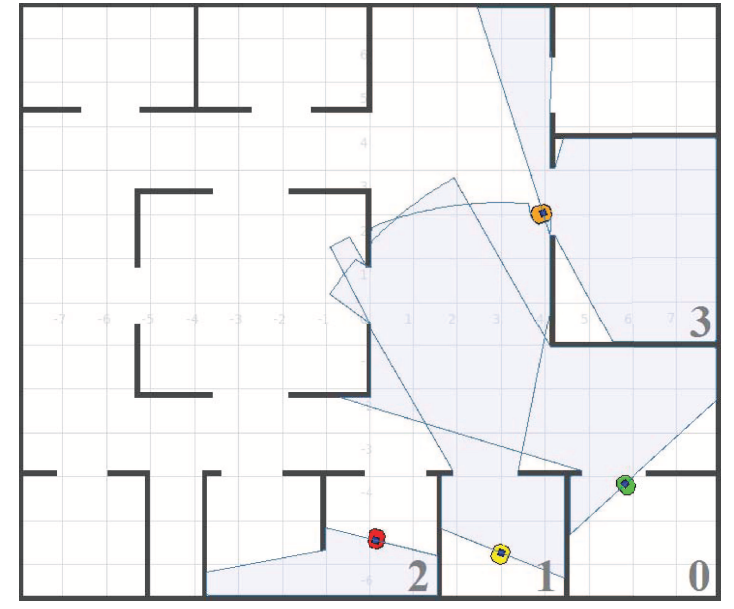

Figure 1: Four robots explore an unknown environment cooperatively. The green robot has completed the exploration of room 0 , is moving to next room. The yellow robot is exploring room 1 . The red robot is exploring room 2 , and the orange robot is moving to room 3 .

theless, this mechanism is ineffectual for large teams with more agents. There is no central planner in the decentralized mechanism. Robots use locally observable informations to make their plans. This mechanism has a good adaptability and strong robustness, but the solutions it got are often sub-optimal.

In this paper, we consider the problem of exploring an unknown indoor environment with a homo- 
geneous team of robots. The larger context of this problem is multi-robot search and rescue in dangerous environments such as fire and explosion. The objective of the research is to explore the entire environment, while minimizing the time needed to complete the overall exploration. In fact, this is also an important prerequisite to better searching and rescuing. Moreover, the realizations of search and rescue are not within the scope of this paper.

This paper presents a novel approach of decentralized dynamic task allocation based on trade rules for groups of robots. This approach is designed to simulate the relationship between buyers and sellers in a business system. The robot which plays the role of buyer applies for a task, and the robot which plays the role of seller is responsible for the assignment of tasks. Due to the context of search and rescue, the following three issues should be considered while designing our trade-based approach:

- Useability, it means that robots are able to implement the proposed approach easily, and use fewer computer system resources.

- Efficiency, it means that robots are able to handle the assignment of tasks with as little communication as possible.

- Robustness, it means that robots are able to make their own decisions correctly in the absence of teammates' information.

In contrast to previous research, especially the well known auction method (Gerkey and Matarić, 2002), our approach uses a application/allocation model unlike the traditional auction/bidding model. The buyer makes a request of task allocation to the seller, we called it the unsolicited bid mechanism. After a period of time, the seller analyzes the received requests, then assigns the tasks to the buyers reasonably. An obvious characteristic of our approach is that it has the ability to assign multiple tasks to multiple robots at a time.

This paper is organized as follows. In Section 2, we give an overview of some related works. Subsequently, we briefly discuss the requirements of indoor exploration in Section 3. Then we present our tradebased approach in Section 4. Finally, we describe the experimental results obtained with our approach in Section 5.

\section{RELATED WORK}

The problem of exploring an unknown environment by a team of mobile robots has received increasing attention in the past few years.
Yamauchi (Yamauchi, 1998) presented a cooperative, decentralized and fault-tolerant multi-robot exploration strategy based on the concept of frontiers. Frontiers are regions on the boundary between open space and unexplored space. In his approach, robots share perceptual information, but maintain separate global maps. Each robot makes its own decisions about where to navigate. Whenever a robot arrives at a new frontier, it sweeps its sensors and constructs a local evidence grid representing its current surroundings. This local grid is integrated with the robots global grid, and also broadcast to all of the other robots. However, different robots may go to explore the same frontier in this system, then the efficiency of exploration will be lowered for this cause.

Burgard, Moors, Fox, Simmons and Thrun (Burgard et al., 2000) designed a coordination component based on the approach of Yamauchi. This component applies a probabilistic method which takes the cost of reaching a frontier and its utility into account simultaneously. The cost is given by the distance of traveling to a frontier (by using value iteration algorithm) and the utility is given by the size of the unexplored area that a robot can cover from this frontier with its sensors. Whenever a frontier is assigned to a robot, the utility of the visible unexplored area of this frontier is reduced to all of the other robots, so that the problem of more than one robot go to the same frontier should no longer appear. However, a central agent is required for this approach. If the central agent fails, the whole system will fail.

Gerkey and Matarić (Gerkey and Matarić, 2002) proposed an auction-based task allocation approach for decentralized multi-robot coordination. The auction proceeds in five steps: task announcement, metric evaluation, bid submission, close of auction and progress monitoring/contract renewal. This strategy has been implemented and tested in a general task allocation system called MURDOCH, which is built upon a principled, resource centric and publish/subscribe communication model. However, their method requires a high amount of data communication. This not only increases the load of system, but also influences the robustness of system.

Zlot, Stentz, Dias and Thayer (Zlot et al., 2002) introduced a market-based task allocation approach for multi-robot exploration. This technique borrows the market architecture which seeks to maximize benefit while minimizing cost, thus aiming to maximize utility. The benefit is information gained by visiting a goal point, the cost is the estimated distance traveled to reach the goal (by using $\mathrm{D}^{*}$ algorithm), then the utility is the difference between the benefit and the cost. Similar to the auction-based strategy, 
the market-based strategy also use the auction/bidding model. However, this approach also requires a high amount of data communication.

Wurm, Stachniss and Burgard (Wurm et al., 2008) proposed a technique for coordinating a team of robotic explorers by using a segmentation of environment. Instead of considering frontiers between unknown and explored areas as target locations, this method takes the structure of the environment into account, and segments the already explored environment based on Voronoi diagram of map, then assigns each robot to a separate segment with a task to explore corresponding area by using the Hungarian algorithm. Their experiments show a significant improvement of the segmentation-based approach compared to a standard frontier-based approach for structured indoor environments. This is favourable for explorations in dangerous environments. However, Their technique still requires a central planning component to communicate with all teammate robots and handle the distribution of tasks.

Marjovi, Nunes, Marques and de Almeida (Marjovi et al., 2009) presented an approach for cooperative multi-robot exploration, fire searching and mapping in an unknown environment, which aims to minimize the overall exploration time while making it possible to localize fire sources in an efficient way. In their approach, a decentralized frontier-based exploration method is used for evaluating the ratio between cost (by using $\mathrm{A}^{*}$ algorithm) and utility to navigate to target waypoints. A potential field method is used for controlling the robots' motion to avoid obstacles, and a triangulation method is used for identifying fire sources. However, the amount of coordination of this approach is limited and a high amount of data communication should be required.

Besides, there are some other approaches developed with various policies (Rekleitis et al., 2000), (Ko et al., 2003), (Stachniss et al., 2009).

In our previous work (Yan et al., 2010), we considered the problem of multi-robot exploration by using separate topological graph. This work aims to solve the waiting situations like congestion or collision during robot motion planning. If all the robots use the same topological graph, then they might follow the same exploration path, so that causes the problem of waiting situations. We proposed an approach based on sampling environment map iteratively to support the efficiently coordinated multi-robot exploration. However, this approach needs a centralized planner to process the map and assign target points to robots. Therefore, in this paper, we study how to design an useable, efficient and robust decentralized system on the one hand, and we still take the structure of envi- ronment to ensure the efficiency of exploration into account on the other hand.

\section{INDOOR EXPLORATION}

Our focus in this paper is on indoor environment exploration. Indoor environments are generally structured environments. For the search and rescue scenario, if the structure of the environment is taken into account, the efficiency of exploration will be greatly improved. Therefore, in our approach, we assume that all robots share a joint occupancy grid map with information about the structure of environment, which is generated from robots' sensor readings. In addition, unlike outdoor exploration, it is difficult for robots localize themselves by using the GPS module in indoor environment, so in our implementation, the robot uses odometry for self-localization.

\section{TRADE-BASED APPROACH}

A business system is mainly made up of buyers and sellers. The relationship between them is known as exchange relation. Buyers can use money to purchase goods or services from sellers, then sellers collect money and sell goods or services to buyers. Our approach in this paper is built on the simulation of this relationship, the model is as follows:

$$
\text { Trade }=<R, M, T, P, C>
$$

where $R$ represents the mobile robots. $M$ represents the whole mission to be completed, which consists of several tasks, $M=\left\{m_{0}, m_{1}, \ldots, m_{n}\right\} . T$ represents the time needed (i.e. the benefit obtained) to complete the whole mission. $P$ signifies the task allocation plan. $C$ signifies the set of cost to complete the whole mission, $C=\left\{c_{0}, c_{1}, \ldots, c_{n}\right\}$. In fact, to each robot, the entire planning contains three steps: role allocation, task allocation and motion planning.

\subsection{Role Allocation}

The role allocation is to solve the problem of which robot should be buyer and which robot should be seller. As far as a homogeneous mobile robot team is concerned, robots themselves have no preference for role. Therefore, a simple and effective way is to number the robots in the team. Robot can broadcast its number when it is not in task, then evaluates the received numbers after a period of time. If there is a number smaller than its own, then the robot will play 
the role of buyer. The details of our implementation are given in Algorithm 1.

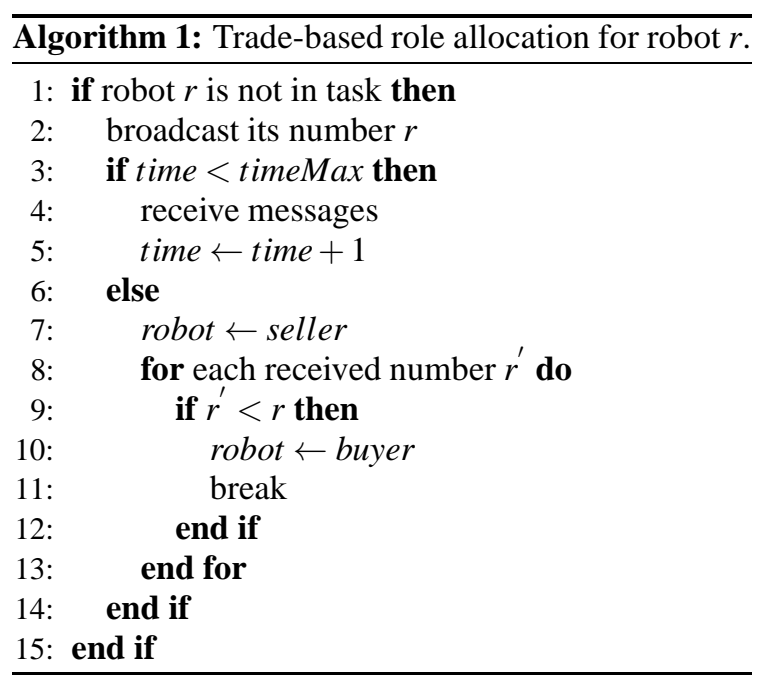

It is worth noting that the difference between buyer robot and seller robot is just in function, and only temporary. The buyer at this moment in a task could become the seller in another task in the next moment.

\subsection{Task Allocation}

Once the idle robot has determined its own role, it should enter the task allocation phase. For the buyer robots, the first thing to do is to choose a task to bid for and estimate the cost required to complete the task. The task is to explore an unknown region which can be identified by topologizing the grid map of the environment (Wurm et al., 2008), (Yan et al., 2010). The robot is capable to extract critical points (Thrun, 1998) for a given map to distinguish corridors, doorways and rooms. The cost metric can be various, such as distance traveled, time taken or energy expended. In this paper, we use the distance traveled as the cost metric. As a result, the robot will calculate the distance between its current position and target position (for example, a critical point) as the estimated task cost by using the wavefront propagation algorithm (LaValle, 2006):

$$
c_{r}^{m}=\text { wavefront }(\operatorname{pos}(r), \operatorname{pos}(m))
$$

where $c_{r}^{m}$ represents the estimated cost $c$ for robot $r$ to complete the task $m$. After completing the cost estimate, the buyer robot should send a purchase request to seller robot for the new task. We called this model the unsolicited bid mechanism. The purchase message mainly contains three information:

$$
\text { pums }_{r}=\{r, m, c\}
$$

where pums $_{r}$ represents the purchase message pums sent by buyer robot $r, r$ in the message means the number of the buyer robot, $m$ signifies the identifier of the task, and $c$ denotes the estimated task cost $c_{r}^{m}$. After the bidding process, the buyer robot will receive a message on whether to get the task from the seller robot or not. If the buyer robot gains the bidden task, it will enter into the motion planning process. Otherwise, it will return to the role allocation process for a new task. A detailed decision making flowchart is given in Figure 2.

For the seller robot, homoplastically, the first step is to select a task and estimate the cost. Then it should collect the purchase message from the buyer robots within a given bidding period. After this period, the seller robot will stop receiving purchase message and evaluate the purchase requisitions, then assign the tasks to each buyer robot for reasonably. The detailed decision making flowchart for seller robot is illustrated in Figure 3. Task allocation is performed by using a greedy algorithm. That is, always selecting the robot with lower estimated cost as the object of task distribution. The implementation details are given in Algorithm 2.

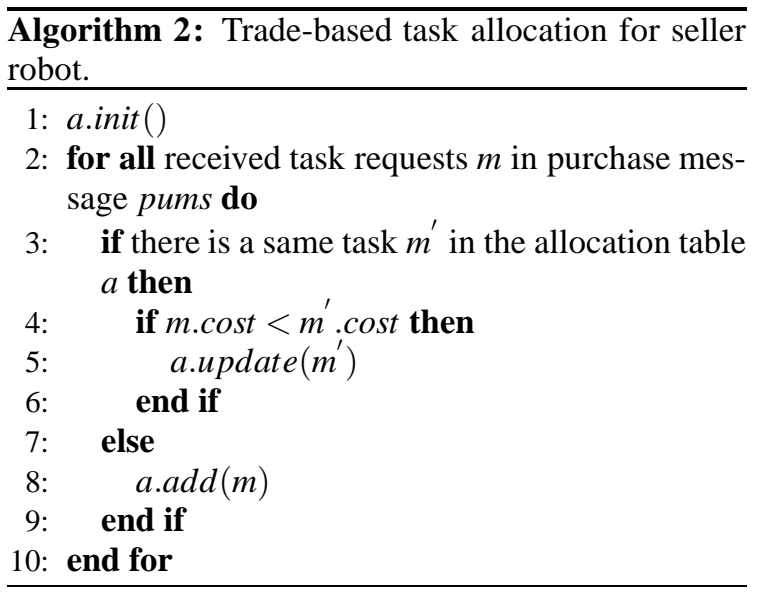

The task allocation message sent to buyer robot mainly includes two information:

$$
\text { tams }_{r}=\{r, m\}
$$

where tams $_{r}$ represents the task allocation message tams sent by seller robot $r, r$ in the message means the number of the seller robot, $m$ signifies the identifier of the task. It is worth noting that, in fact, the seller robot itself is also the object of task allocation.

The robot which has completed a task will update its task list, and also broadcast to all teammates. 


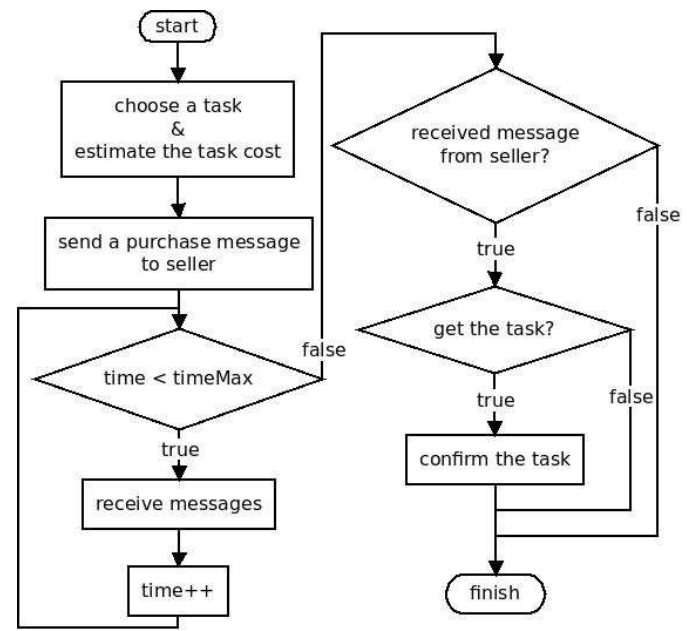

Figure 2: Decision making flowchart for buyer robot.

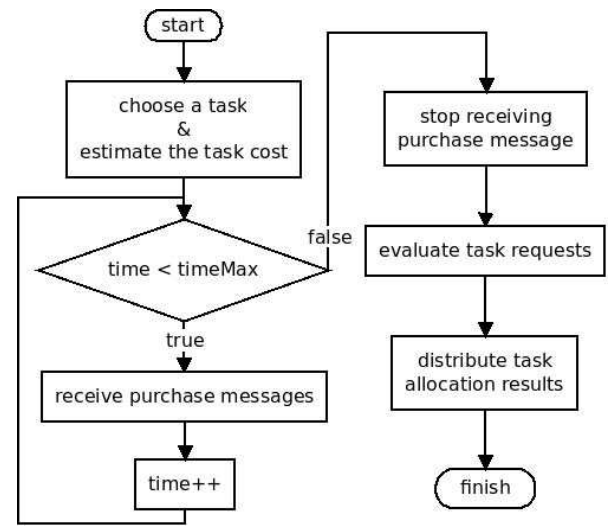

Figure 3: Decision making flowchart for seller robot.

\subsection{Motion Planning}

The motion planning process is in charge of the implementation of task, i.e. the exploration of the unknown region. In our approach, the mobile robots will share an occupancy grid map with respect to the structure of the environment during their exploration mission. This map is used for path planning and obstacle avoidance in real time. Consequently, we use the wavefront propagation algorithm (LaValle, 2006) for global path planning and the vector field histogram algorithm (Ulrich and Borenstein, 1998) for goal seeking and local obstacle avoidance.

\section{EXPERIMENTS}

Our approach has been implemented and evaluated in Stage (Gerkey et al., 2003) a 2.5D multiple-robot simulator. The simulation experiments were conducted by using a group of Pioneer 2-DX robot equipped with a laser range finder which can provide 361 samples with 180 degrees field of view and a maximum range of 8 meters. Each robot can localize itself based on an abstract localization device which models the implementation of SLAM. The ratio between realworld time and simulation time is about 1:3. All experiments reported in this paper were carried out on a system with an Intel Core 2 Duo E8400 3.00GHz processor, an Intel Q43 Express chipset and two DDR2 $800 \mathrm{MHz} 1024 \mathrm{MB}$ dual channel memory.
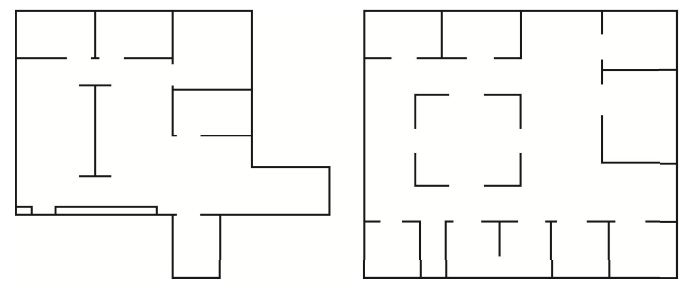

Figure 4: Two environment maps used in our simulation: map A (left) and map B (right).

To evaluate our trade-based approach, we used a different number of robots to conduct several experiments in various environments. We also compared our approach to a centralized approach based on the Hungarian method (Kuhn, 1955) in which the task allocation is done by a central planner. Figure 4 depicts two maps used in our simulation which are enclosed spaces with 14 meters long and 16 meters wide. Both of them are indoor structured environments. The screenshot of our implementation can be seen in Figure 1, and the results of our experiments are given in Table 1 and Table 2.

Table 1 shows a comparison of the results between our trade-based approach (decentralized) and the Hungarian-based approach (centralized) in map A with the context of measuring the exploration runtime. Each set of data in the table contains an average time (in seconds) of 10 runs and its standard deviation in parentheses. The Hungarian method is an efficient way to find an optimal solution for a task assignment problem. In our experiment, this procedure is used as a reference for measuring the performance of our proposed method. Table 1 indicates that, the difference on completion time between trade-based exploration and Hungarian-based exploration is $6.6 \%$ (4.3 seconds) for 2 robots, $19.4 \%$ (8.9 seconds) for 3 robots, and $3.9 \%$ (1.8 seconds) for 4 robots respectively.

Table 2 shows the results for map B. The runtime difference is $6.3 \%$ ( 4.5 seconds) for 3 robots and $7.4 \%$ (4.6 seconds) for 4 robots respectively. Moreover, we observed that, our trade-based approach gained $14.2 \%$ time (13.9 seconds) compared to the Hungarian-based approach with 2 robots. It reflects 
Table 1: Exploration Runtime Comparison for Map A.

\begin{tabular}{c||c|c}
\hline & $\begin{array}{c}\text { Hungarian-based } \\
\text { exploration }\end{array}$ & $\begin{array}{c}\text { Trade-based } \\
\text { exploration }\end{array}$ \\
\hline \hline 2 robots & $65.20 \mathrm{~s}(1.166)$ & $69.50 \mathrm{~s}(0.806)$ \\
\hline 3 robots & $45.90 \mathrm{~s}(0.538)$ & $54.80 \mathrm{~s}(1.887)$ \\
\hline 4 robots & $45.90 \mathrm{~s}(7.120)$ & $47.70 \mathrm{~s}(0.458)$ \\
\hline
\end{tabular}

Table 2: Exploration Runtime Comparison for Map B.

\begin{tabular}{c||c|c}
\hline & $\begin{array}{c}\text { Hungarian-based } \\
\text { exploration }\end{array}$ & $\begin{array}{c}\text { Trade-based } \\
\text { exploration }\end{array}$ \\
\hline \hline 2 robots & $112.0 \mathrm{~s}(3.317)$ & $98.10 \mathrm{~s}(1.300)$ \\
\hline 3 robots & $71.70 \mathrm{~s}(1.487)$ & $76.20 \mathrm{~s}(3.027)$ \\
\hline 4 robots & $61.80 \mathrm{~s}(1.249)$ & $66.40 \mathrm{~s}(1.020)$ \\
\hline
\end{tabular}

NP-hard of multi-robot exploration from one side.

With Table 1 and Table 2, we found that, although our trade-based approach requires more time compared to the Hungarian-based approach in most cases, the difference is quite acceptable. Consequently, the experimental results still reflect a good performance of our proposed approach.

Another experiment was conducted to evaluate the robustness of our method. The point is to test the effect of decision making of a robot under uncertainty caused by information loss. The motivations of the loss may be damage of robot components, communication obstacles (distance), or difficult circumstances. For a coordinated robots team with a decentralized decision making system, each robot in the team need to exchange information with its teammates. The problem of information loss will influence the decision performance. Table 3 and Table 4 show the robustness testing results of our proposed method in map A and map B respectively, in which we varied the probability of information loss with 10\%, 30\% and 50\%. Each set of data in the tables contains an average time (in seconds) of 10 runs.

Table 3 shows that, in map A: Under 10\% information loss, the system keeps a good performance, the exploration runtimes are prolonged but still close to the normal results, the differences between them are 0.9 seconds ( 2 robots), 1.4 seconds ( 3 robots) and 3.1 seconds (4 robots). Under $30 \%$ information loss, the performance of system was lowered, the exploration runtimes are prolonged by the undesirable effect of the loss. Compared with the normal results, the differences are 15.0 seconds (2 robots), 8.2 seconds (3 robots) and 12.0 seconds (4 robots). Under 50\% information loss, the performance of system keeps lowering, and the differences between the exploration runtimes and the normal results are 15.6 seconds ( 2
Table 3: Robustness Testing with Map A.

\begin{tabular}{c||c|c|c}
\hline \multicolumn{1}{c||}{} & \multicolumn{3}{c}{ Information loss probability } \\
\cline { 2 - 4 } & $\mathbf{1 0 \%}$ & $\mathbf{3 0 \%}$ & $\mathbf{5 0 \%}$ \\
\hline \hline 2 robots & $70.40 \mathrm{~s}$ & $84.50 \mathrm{~s}$ & $85.10 \mathrm{~s}$ \\
\hline 3 robots & $56.20 \mathrm{~s}$ & $63.00 \mathrm{~s}$ & $76.80 \mathrm{~s}$ \\
\hline 4 robots & $50.80 \mathrm{~s}$ & $59.70 \mathrm{~s}$ & $73.90 \mathrm{~s}$ \\
\hline
\end{tabular}

Table 4: Robustness Testing with Map B.

\begin{tabular}{c||c|c|c}
\hline \multicolumn{1}{c||}{} & \multicolumn{3}{c}{ Information loss probability } \\
\cline { 2 - 4 } & $\mathbf{1 0 \%}$ & $\mathbf{3 0 \%}$ & $\mathbf{5 0 \%}$ \\
\hline \hline 2 robots & $100.3 \mathrm{~s}$ & $141.3 \mathrm{~s}$ & $155.2 \mathrm{~s}$ \\
\hline 3 robots & $82.00 \mathrm{~s}$ & $102.5 \mathrm{~s}$ & $124.6 \mathrm{~s}$ \\
\hline 4 robots & $67.00 \mathrm{~s}$ & $83.00 \mathrm{~s}$ & $92.80 \mathrm{~s}$ \\
\hline
\end{tabular}

robots), 22.9 seconds (3 robots) and 26.2 seconds (4 robots).

Table 4 shows that, for map B: Under $10 \%$ information loss, the differences are 2.2 seconds ( 2 robots), 5.8 seconds (3 robots) and 0.6 seconds (4 robots) compared with normal results. Under $30 \%$ information loss, the differences are 43.2 seconds (2 robots), 26.3 seconds (3 robots) and 16.6 seconds (4 robots). Under $50 \%$ information loss, the differences are 57.1 seconds ( 2 robots), 48.4 seconds (3 robots) and 26.4 seconds (4 robots).

With Table 3 and Table 4, we found that, multirobot coordination depends on the information exchange between teammate robots, the exploration runtimes are prolonged for the probability of information loss. Moreover, the corresponding increments are considered acceptable in our experiments. This demonstrates a good robustness of our proposed trade-based approach. In fact, our approach still work in extreme cases (zero communication), but the efficiency is lower.

\section{CONCLUSIONS}

In this paper, we presented a novel task allocation approach under decentralized coordination for multirobot exploration. The basic thought of the proposed approach is to simulate the relationship between buyers and sellers in a business system, and dynamically allocate the task by using an unsolicited bid mechanism. Typically, the procedure goes like this: At first, we should determine the role of the robots, i.e. buyer or seller, this step is known as role allocation. Secondly, the buyer robot should choose a task to bid for, then the seller robot will assign the tasks to each buyer 
reasonably, this step is known as task allocation. An iterative greedy method is applied in our implementation for the step of task allocation.

For the coordinated decision making in decentralized multi-robot system, robots should make their plans according to the local observable information with limited communication. This paper takes the problem of multi-robot search and rescue in dangerous environments as the background. The conception of our trade-based approach can meet the system's requirements of useability, efficiency and robustness. The first experiment we have conducted is designed to evaluate the performance of our proposed method, and the second is designed to test the robustness (in the case of information loss). The experimental results demonstrate that, our trade-based approach has a good efficiency for decentralized multi-robot exploration.

However, the actual limitation of the proposed approach is that it can not guarantee the global optimal solution will be found, i.e. the task allocation plan for the whole mission is sometimes not optimal. Future work will improve the step of task allocation. An implementable idea is that the buyer robot can send the purchase request to the seller robot for several tasks, then seller robot will evaluate the purchase requisitions and assign the tasks with a more advanced algorithm (i.e. improve Algorithm 2).

\section{REFERENCES}

Burgard, W., Moors, M., Fox, D., Simmons, R., and Thrun, S. (2000). Collaborative multi-robot exploration. In Proceedings of the 2000 IEEE International Conference on Robotics and Automation (ICRA'O0), pages 476-481, San Francisco, CA, USA.

Cao, Y. U., Fukunaga, A. S., and Kahng, A. (1997). Cooperative mobile robotics: Antecedents and directions. Autonomous Robots, 4(1):7-27.

Dudek, G., Jenkin, M. R. M., Milios, E., and Wilkes, D. (1996). A taxonomy for multi-agent robotics. Autonomous Robots, 3(4):375-397.

Gerkey, B. P. and Matarić, M. J. (2002). Sold!: Auction methods for multirobot coordination. IEEE Transactions on Robotics and Automation, 18(5):758-768.

Gerkey, B. P. and Matarić, M. J. (2004). A formal analysis and taxonomy of task allocation in multi-robot systems. The International Journal of Robotics Research, 23(9):939-954.

Gerkey, B. P., Vaughan, R. T., and Howard, A. (2003). The player/stage project: Tools for multi-robot and distributed sensor systems. In Proceedings of the 11th International Conference on Advanced Robotics (ICAR'03), pages 317-323, Coimbra, Portugal.
Ko, J., Stewart, B., Fox, D., Konolige, K., and Limketkai, B. (2003). A practical, decision-theoretic approach to multi-robot mapping and exploration. In Proceedings of the 2003 IEEE/RSJ International Conference on Intelligent Robots and Systems (IROS'03), pages 3232-3238, Las Vegas, NV, USA.

Kuhn, H. W. (1955). The hungarian method for the assignment problem. Naval Research Logistics Quarterly, 2(1):83-97.

LaValle, S. M. (2006). Planning Algorithms. Cambridge University Press.

Marjovi, A., Nunes, J. G., Marques, L., and de Almeida, A. (2009). Multi-robot exploration and fire searching. In Proceedings of the 2009 IEEE/RSJ International Conference on Intelligent Robots and Systems (IROS'09), pages 1929-1934, St. Louis, MO, USA.

Rekleitis, I. M., Dudek, G., and Milios, E. E. (2000). Multirobot collaboration for robust exploration. In Proceedings of the 2000 IEEE International Conference on Robotics and Automation (ICRA'O0), pages 3164 3169, San Francisco, CA, USA.

Stachniss, C., Mozos, Ó. M., and Burgard, W. (2009). Efficient exploration of unknown indoor environments using a team of mobile robots. Annals of Mathematics and Artificial Intelligence, 52(2):205ff.

Thrun, S. (1998). Learning metric-topological maps for indoor mobile robot navigation. Artificial Intelligence, 99(1):21-71.

Ulrich, I. and Borenstein, J. (1998). VFH+: Reliable obstacle avoidance for fast mobile robots. In Proceedings of the 1998 IEEE International Conference on Robotics and Automation (ICRA'98), pages 1572-1577, Leuven, Belgium.

Wurm, K. M., Stachniss, C., and Burgard, W. (2008). Coordinated multi-robot exploration using a segmentation of the environment. In Proceedings of the 2008 IEEE/RSJ International Conference on Intelligent Robots and Systems (IROS'08), pages 1160 1165, Nice, France.

Yamauchi, B. (1998). Frontier-based exploration using multiple robots. In Proceedings of the 2nd International Conference on Autonomous Agents (Agents'98), pages 47-53, Minneapolis, MN, USA.

Yan, Z., Jouandeau, N., and Ali Cherif, A. (2010). Sampling-based multi-robot exploration. In Proceedings of the Joint 41th International Symposium on Robotics and 6th German Conference on Robotics (ISR/ROBOTIK 2010), pages 44-49, Munich, Germany.

Zlot, R., Stentz, A. T., Dias, M. B., and Thayer, S. (2002). Multi-robot exploration controlled by a market economy. In Proceedings of the 2002 IEEE International Conference on Robotics and Automation (ICRA'02), pages 3016-2023, Washington, DC, USA. 